\title{
The Paula method and the pelvic floor: comment
}

\author{
Michal Liebergall-Wischnitzer
}

Published online: 12 April 2011

(C) The International Urogynecological Association 2011

\section{Dear Editor}

I was pleased to find two research articles on the Paula method written by Bo et al. [1] and Resende et al. [2]. I agree with the authors that pelvic floor muscle training (PFMT) is effective and should be recommended as level A evidence for pelvic floor dysfunction. However, I would like to comment on the present research and explain some of the issues which I believe are not clearly understood.

Both researchers chose reliable tools: ultrasound and electromyography. However, the validity is questionable. Are these tools suitable to assess the Paula method? PFM strength is not a desirable outcome of Paula exercises, unlike in simple PFMT. So, despite having used reliable tools, both studies missed the main goal of the Paula method and chose to measure an inappropriate outcome.

The researchers each picked either one [1] or several [2] exercises and measured PFM strength results immediately following exercise [1] or $40 \mathrm{~min}$ thereafter [2]. This is a very limited time for Paula method intervention and unacceptable for any other method of exercise. Is it logical to assess yoga or tai chi after only one session? As in other physical activity methods, Paula exercise does not provide immediate results. Contracting the eyes/mouth and the PFM co-contraction is not a reflex; training is required. After a training period, the trainee begins to feel that these muscles can work together and naturally integrate with PFMT.

\footnotetext{
M. Liebergall-Wischnitzer $(\bowtie)$

Henrietta Szold School of Nursing in the Faculty of Medicine, Hadassah/Hebrew University Medical Center,

POB 12000, Jerusalem 91120, Israel

e-mail: michall2@hadassah.org.il
}

The Paula instructor gave the trainee an "open order," e.g., "open and close the mouth." Other muscles may or may not join in, depending on how the trainee feels. The cocontraction can be fast or it may take a long time. The theory behind the method is not as yet clear, as has been mentioned in the literature. Translational research is acceptable, even in situations where the theory behind it is not fully understood but can be found in the medical literature.

Any research in the field needs to involve a certified Paula instructor who has an in-depth understanding of the method, as was done in a recent publication in this journal. There, a comparison was made between PFMT and Pilates [3], using certified Pilates instructors (with a timeframe of 24 weeks), in contrast to the present research $[1,2]$.

Both groups [1,2] perceive the Paula method as "PFMT plus" and not as an independent method, as it should be.

\section{References}

1. Bo K, Hilde G, Staer-Jensen J, Braekken IH (2010) Can the Paula method facilitate co-contraction of the pelvic floor muscles? A 4D ultrasound study. Int Urogynecol J Pelvic Floor Dysfunct. doi:10.1007/s00192-010-1317-8, published online 23 November 2010

2. Resende AP, Zanetti MR, Petricelli CD, Castro RA, Alexandre SM, Nakamura MU (2010) Effects of the Paula method in electromyographic activation of the pelvic floor: a comparative study. Int Urogynecol J Pelvic Floor Dysfunct. doi:10.1007/s00192-0101331-x, published online 23 November 2010

3. Culligan PJ, Scherer J, Dyer K, Priestley JL, Guingon-White G, Delvecchio D, Vangeli M (2010) A randomized clinical trial comparing pelvic floor muscle training to a Pilates exercise program for improving pelvic muscle strength. Int Urogynecol J Pelvic Floor Dysfunct 21(4):401-408 